\title{
Visualization of Magnetic Nanofibers Using Magnetic Particle Imaging
}

\author{
Kenya Murase, Atsushi Mimura, Natsuo Banura, Kohei Nishimoto, Hiroshige Takata \\ Department of Medical Physics and Engineering, Division of Medical Technology and Science, Faculty of Health \\ Science, Graduate School of Medicine, Osaka University, Osaka, Japan \\ Email: murase@sahs.med.osaka-u.ac.jp
}

Received 30 April 2015; accepted 30 May 2015; published 2 June 2015

Copyright ( 2015 by authors and Scientific Research Publishing Inc.

This work is licensed under the Creative Commons Attribution International License (CC BY).

http://creativecommons.org/licenses/by/4.0/

(c) $\underset{\mathrm{EY}}{\mathrm{EY}}$ Open Access

\section{Abstract}

Purpose: Magnetic nanofibers (MNFs) are nanofibers impregnated with magnetic nanoparticles (MNPs). Magnetic particle imaging (MPI) is a recently introduced imaging method that allows imaging of the spatial distribution of MNPs. The purpose of this study was to develop MNFs and to investigate the feasibility of visualizing them using MPI and of heating them using an alternating magnetic field (AMF). Materials and Methods: First, chitosan nanofibers were cross-linked with glutaraldehyde vapor in a sealed vial for 24 hours. Next, they were mixed with various concentrations of MNPs and the mixture was stirred for 1 hour using a magnetic stirrer. After the mixture was refrigerated at $-80^{\circ} \mathrm{C}$ for 24 hours, it was freeze-dried for 24 hours. The morphology of the resultant MNFs was characterized by scanning electron microscopy, and the magnetic properties were measured using a vibrating sample magnetometer. After these measurements, we imaged the MNFs using our MPI scanner, and investigated the correlation between the pixel values of the MPI image and the concentration of MNPs or the number of MNF sheets. We also heated the MNFs using AMF, and measured the temperature rise using an infrared thermometer. Results: The MNFs were successfully visualized using our MPI scanner, and the pixel values of the MPI image showed excellent correlation with the concentration of MNPs $(r=0.992)$ and the number of MNF sheets $(r=0.997)$. A significant temperature rise was observed under AMF, and the initial slope of the time-dependent temperature rise showed excellent correlation with the concentration of MNPs $(r=0.994)$ and the number of MNF sheets $(r=0.979)$. Conclusion: The MNFs developed in this study can be visualized using MPI and can be applied to magnetic hyperthermia. They will be useful in biomedicine including cancer therapy and tissue regeneration.

\section{Keywords}

Magnetic Nanofibers, Magnetic Particle Imaging, Magnetic Nanoparticles, Magnetic Hyperthermia 


\section{Introduction}

The use of scaffolds in tissue engineering is constantly increasing, because these materials have become fundamental tools to help the body rebuild damaged or diseased tissues [1]. Of the scaffolds, the magnetic scaffold has attracted increasing attention because it can attract and take up angiogenic proteins such as growth factors (GFs) or other bio-agents such as stem cells in vivo via a driving magnetic force. This concept involves the use of magnetic nanoparticles (MNPs) which are functionalized with GFs or other bio-agents to be taken up by the magnetic scaffold [1]. These functionalized MNPs act as carriers transporting the bio-agents towards and inside the magnetic scaffold under the effect of a magnetic field [1].

Some mechanical stimulators such as stress and strain have been shown to enhance bone regeneration and fracture healing [2]. Besides these mechanical stimuli, magnetic stimulation originating from static or alternating magnetic fields has also attracted researchers' interest [3]. Weak magnetic or pulse electromagnetic fields of Gauss order have been reported to be effective for promoting bone fracture healing and spinal fusion [3]. Strong static magnetic fields of 5 to 10 Tesla have also been reported to potently regulate the orientation of matrix proteins and cells in vitro and in vivo [3]. Meng et al. [3] reported that the magnetic scaffold under a static magnetic field enhanced osteogenesis and provided a novel strategy for scaffold-guided bone repair.

It is well known that MNPs can induce heat when placed in an alternating magnetic field. On the basis of this behavior, the use of bone cement containing MNPs has been proposed for the hyperthermic treatment of bone tumors [4]. Matsumine et al. [5] prepared calcium phosphate cement containing $\mathrm{Fe}_{3} \mathrm{O}_{4}$ and conducted clinical trials to treat patients with metastatic bone lesions by hyperthermia, and reported that the patients treated by hyperthermia showed better radiographic outcomes than those treated by palliative operation.

Magnetic nanofibers (MNFs) are a magnetic scaffold, comprising nanofibers impregnated with MNPs. MNFs have huge potential in biomedical applications such as cancer therapy including hyperthermia [6] and tissue regeneration [5]. Visualization of MNFs as well as accurate knowledge about the quantity of MNPs within the MNFs is crucial for effective and safe treatment planning of cancer therapy or tissue regeneration using them [5] [6]. Thus, the development of a readily available, noninvasive, and quantitative imaging technique will be necessary if cancer therapy or tissue regeneration using MNFs is to succeed.

Recently, a new imaging method called magnetic particle imaging (MPI) has been introduced [7]. MPI allows imaging of the spatial distribution of MNPs with high sensitivity, spatial resolution, and imaging speed [7]. MPI uses the nonlinear response of MNPs to detect their presence in an alternating magnetic field, which is referred to here as the drive magnetic field. Spatial encoding is accomplished by saturating the MNPs over most of the imaged region using a static magnetic field (selection magnetic field), except in the vicinity of a special position called the field-free point [7] or field-free line [8]. We have developed a system for MPI with a field-free-line encoding scheme, in which the field-free line is generated using two opposing neodymium magnets, and transverse images are reconstructed from the third-harmonic signals received by a gradiometer coil using the maximum likelihood-expectation maximization (ML-EM) algorithm [9] [10].

The purpose of this study was to develop MNFs and to investigate the feasibility of visualizing them using MPI and of utilizing them as a source of magnetic hyperthermia.

\section{Materials and Methods}

\subsection{Preparation of Magnetic Nanofibers}

In this study, we used chitosan nanofibers (Sugino Machine Co., Ltd., Uozu, Toyama, Japan) as the source of MNFs, prepared from dry chitosan powder using a high-pressure water jet system [11]. First, chitosan nanofibers were cross-linked with glutaraldehyde (Wako Pure Chemical Industries Co., Ltd., Osaka, Osaka, Japan) vapor in a sealed vial for 24 hours to strengthen the structure of nanofibers and to gain degradation resistance (Figure 1(a)). Next, they were mixed with M-300 (magnetite, $\mathrm{Fe}_{3} \mathrm{O}_{4}$ ) (Sigma Hi-chemical Inc., Chigasaki, Kanagawa, Japan) [12] at various concentrations $(0,25,50,100$, and $150 \mathrm{mM} \mathrm{Fe})$. The mixture was stirred at 450 rpm for 1 hour using a magnetic stirrer (Figure $1(\mathrm{~b})$ ). After the mixture was stirred, it was refrigerated at $-80^{\circ} \mathrm{C}$ for 24 hours, and then it was freeze-dried at $-50^{\circ} \mathrm{C}$ and 1.8 mbar for 24 hours using a freeze dryer (Free Zone 2.5, Labconco Co., Kansas, MO, USA) (Figure 1(c)).

\subsection{System for Magnetic Particle Imaging}

Figure 2 shows a photograph of our MPI scanner. The details of our MPI system are described in our previous 


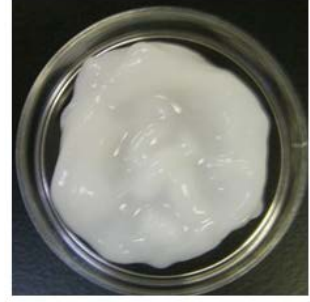

(a)

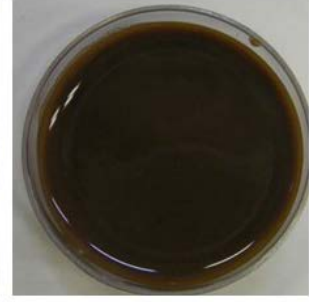

(b)

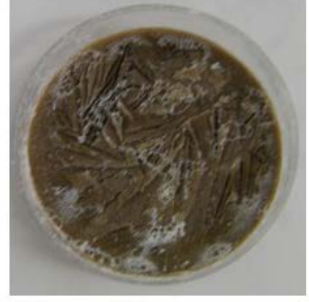

(c)

Figure 1. (a) Chitosan nanofibers cross-linked with glutaraldehyde vapor in a sealed vial for 24 hours, (b) magnetic nanofibers (MNFs) fabricated by mixing the cross-linked chitosan nanofibers with magnetic nanoparticles (MNPs), and (c) MNFs freeze-dried for 24 hours.

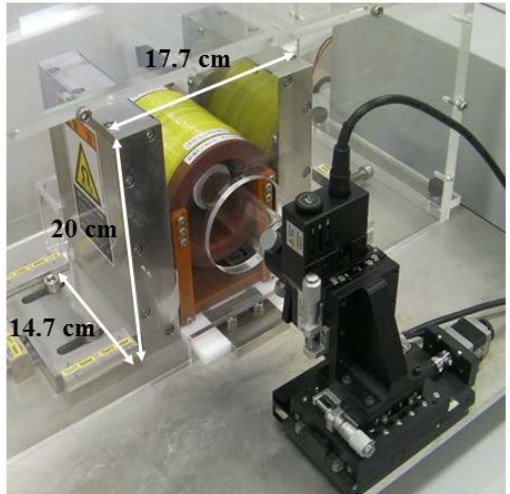

Figure 2. Photograph of our scanner for magnetic particle imaging (MPI).

paper [9] [10]. In brief, the field-free line was generated by two opposing neodymium magnets. The drive magnetic field was generated using an excitation coil (solenoid coil $100 \mathrm{~mm}$ in length, $80 \mathrm{~mm}$ in inner diameter, and $110 \mathrm{~mm}$ in outer diameter). AC power was supplied to the excitation coil by a programmable power supply (EC1000S, NF Co., Yokohama, Kanagawa, Japan), and was controlled using a sinusoidal wave generated by a digital function generator (DF1906, NF Co., Yokohama, Kanagawa, Japan). The frequency of the drive magnetic field was $400 \mathrm{~Hz}$, and the peak-to-peak strength of the drive magnetic field was $20 \mathrm{mT}$. The MNP-generated signal was detected by a gradiometer coil $(50 \mathrm{~mm}$ in length, $35 \mathrm{~mm}$ in inner diameter, and $40 \mathrm{~mm}$ in outer diameter) and the third-harmonic signal was extracted using a preamplifier (T-AMP03HC, Turtle Industry Co., Tsuchiura, Ibaragi, Japan) and a lock-in amplifier (LI5640, NF Co., Yokohama, Kanagawa, Japan). The output of the lock-in amplifier was converted to digital data using a personal computer connected to a multifunction data acquisition device with a universal serial bus port (USB-6212, National Instruments Co., Austin, TX, USA). The sampling time was taken as $10 \mathrm{~ms}$. When measuring signals using the gradiometer coil, a sample was placed $12.5 \mathrm{~mm}$ (i.e., one quarter of the coil length) from the center of the gradiometer coil and the coil, including the sample, was moved such that the center of the sample coincided with the position of the field-free line. The selection magnetic field was generated by two opposing neodymium magnets (Neomax Engineering Co., Takasaki, Gunma, Japan). The field-free line can be generated at the center of the two neodymium magnets.

To acquire projection data for image reconstruction, both the sample and the receiving coil were automatically rotated around the z-axis over $180^{\circ}$ in steps of $5^{\circ}$ and translated in the $\mathrm{x}$-direction from $-16 \mathrm{~mm}$ to $16 \mathrm{~mm}$ in steps of $1 \mathrm{~mm}$, using an XYZ-axes rotary stage (HPS80-50X-M5, Sigma Koki Co., Sumida, Tokyo, Japan), which was controlled using LabVIEW (National Instruments Co., Austin, TX, USA). Data acquisition took about $12 \mathrm{~min}$. Each projection data set was then transformed into 64 bins by linear interpolation. Both the inhomogeneous sensitivity of the receiving coil and feed through interference were corrected using the method described in [13]. Transverse images were reconstructed from the projection data using the ML-EM algorithm over 15 iterations, in which the initial concentration of MNPs was assumed to be uniform [9] [10]. 


\subsection{System for Magnetic Hyperthermia}

The details of our system for magnetic hyperthermia are described in our previous paper [14]. In brief, an alternating magnetic field was generated with use of an external coil comprising 19-turned loops $(6.5 \mathrm{~cm}$ in diameter and $10 \mathrm{~cm}$ in length) of copper pipe ( $5 \mathrm{~mm}$ in diameter) and was cooled by water to ensure a constant temperature and impedance. The coil was connected to a power supply (T162-5723BHE, Thamway Co., Fuji, Shizuoka, Japan) through an impedance tuner (T020-5723AHE, Thamway Co., Fuji, Shizuoka, Japan). This system induced an alternating magnetic field with a maximum amplitude of $3.7 \mathrm{kA} / \mathrm{m}$ at an output power of $500 \mathrm{~W}$. The amplitude of the alternating magnetic field can be controlled by changing the output of the power supply. In this study, the frequency and amplitude of the alternating magnetic field were taken as $600 \mathrm{kHz}$ and $3.1 \mathrm{kA} / \mathrm{m}$, respectively.

\subsection{Evaluation of Magnetic Nanofibers}

The morphology of the resultant MNFs was characterized by a scanning electron microscope (SEM) (Hitachi High-Technologies Co., Ltd., Chiyoda, Tokyo, Japan). The magnetic properties were measured using a vibrating sample magnetometer (VSM) (Riken Denshi Co., Ltd., Meguro, Tokyo, Japan). After these measurements, we imaged the MNFs (10 $\mathrm{mm}$ in diameter and $2 \mathrm{~mm}$ in thickness) with various concentrations of MNPs $(0,25,50$, 100 , and $150 \mathrm{mM} \mathrm{Fe}$ ) and various numbers of MNF sheets with a concentration of $100 \mathrm{mM} \mathrm{Fe}(1,2$, and 3 sheets) using our MPI scanner [9] [10]. We then calculated the average MPI value by drawing a circular region of interest (ROI) with a diameter of $10 \mathrm{~mm}$ on the MPI image. In this study, we defined an MPI value as the pixel value of the transverse image reconstructed from the third-harmonic signals. We then investigated the correlation of the average MPI value with the concentration of MNPs and the number of MNF sheets.

We also heated the MNFs for 10 min using our apparatus for magnetic hyperthermia [14] at a frequency of $600 \mathrm{kHz}$ and an amplitude of $3.1 \mathrm{kA} / \mathrm{m}$, and measured the time course of the temperature rise during heating using an infrared thermometer (FLIR E4, FLIR Systems Inc., Wilsonville, OR, USA) every 30 s during the first 2 min and every $1 \mathrm{~min}$ during the next $8 \mathrm{~min}$ after the beginning of heating. The time-dependent temperature rise $(\Delta T(t))$ thus measured was fitted by use of the phenomenological Box-Lucas equation given by [15]

$$
\Delta T(t)=T(t)-T(0)=A\left(1-\mathrm{e}^{-B t}\right)
$$

where $T(t)$ and $T(0)$ are the temperatures at time $t$ and 0 , respectively, and $A$ and $B$ are constants. This equation is often used to describe the heating of MNPs using an alternating magnetic field [16]. The product of the fitting parameters, $A \times B$, is equivalent to the initial slope of the time-dependent temperature rise $(\Delta T / \Delta t)_{0}$, i.e.,

$$
\left(\frac{\Delta T}{\Delta t}\right)_{0}=A \times B
$$

We investigated the correlation of $(\Delta T / \Delta t)_{0}$ with the concentration of MNPs and the number of MNF sheets.

\subsection{Magnetic Particle Imaging of Magnetic Nanofibers Implanted in the Bone}

To simulate therapy of a bone tumor or bone regeneration using MNFs, we created a hole with a diameter of 4 $\mathrm{mm}$ in the bone of a chicken, and implanted MNFs with a concentration of $150 \mathrm{mM}$ Fe into the hole. To investigate whether MNFs implanted in the bone can be visualized by MPI, the bone implanted with the MNFs was imaged using our MPI scanner [9] [10]. After the MPI study, X-ray CT images were obtained using a 4-row multi-slice CT scanner (Asteion, Toshiba Medical Systems Co., Odawara, Tochigi, Japan) with a tube voltage of $120 \mathrm{kV}$ and a tube current of $210 \mathrm{~mA}$. The MPI image was co-registered with the X-ray CT image using the parameters for magnification and rotation, which were obtained using a phantom with 3 point sources with a diameter of $0.5 \mathrm{~mm}$ and filled with $100 \mathrm{mM}$ MNPs.

\subsection{Statistical Analysis}

The correlations of the average MPI value with the concentration of MNPs and the number of MNF sheets were analyzed using linear regression analysis and the correlation coefficients and regression equations were calcu- 
lated. The correlations of $(\Delta T / \Delta t)_{0}$ with the concentration of MNPs and the number of MNF sheets were also analyzed using linear regression analysis. A $P$ value less than 0.05 was considered statistically significant.

\section{Results}

Figure 3 shows the SEM images of MNFs. As shown in Figure 3, chitosan nanofibers were seen when the concentration of MNPs was low. In contrast, globular aggregations like cocoons were observed when the concentration of MNPs was high.

Figure 4 shows the magnetization of the MNFs with various concentrations of MNPs, which was measured using VSM. As shown in Figure 4, the MNFs had a nonlinear response to a magnetic field, and the saturation magnetization increased with increasing concentration of MNPs.

Figure 5 shows the MPI images of MNFs (upper row) and the thermal images obtained by an infrared thermometer $10 \mathrm{~min}$ after the start of magnetic hyperthermia (lower row), with various concentrations of MNPs. Figure 6 shows the case when the number of MNF sheets was varied. As shown in the upper row of Figure 5, the pixel value of the MPI image increased with increasing concentration of MNPs. It also increased with increasing number of MNF sheets as shown in the upper row of Figure 6. The temperature of MNFs 10 min after

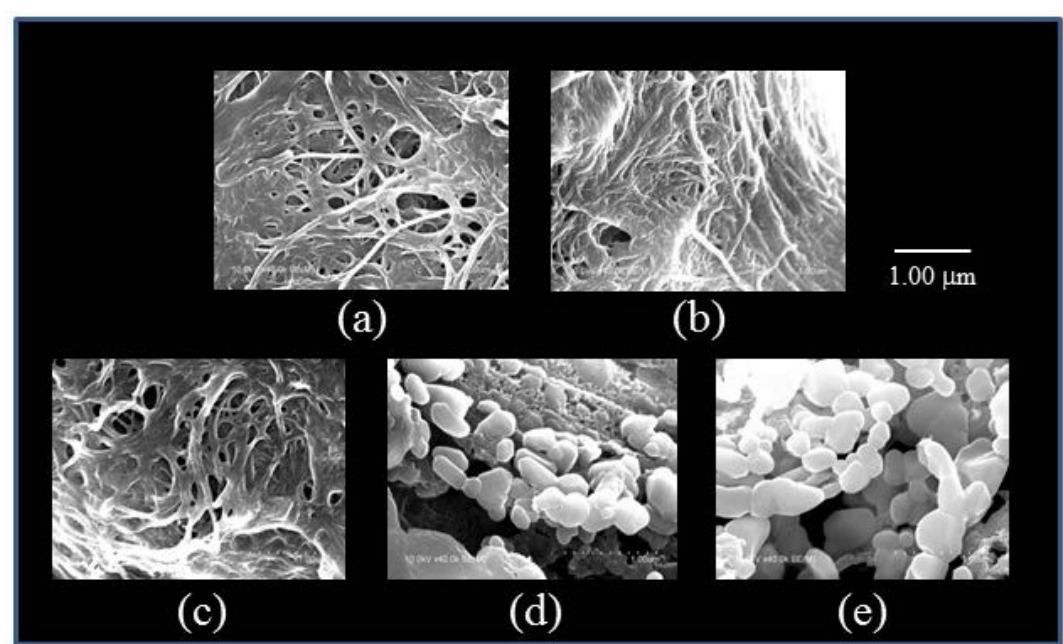

Figure 3. Scanning electron microscope (SEM) images of the MNFs with various concentrations of MNPs. (a) $0 \mathrm{mM} \mathrm{Fe}$, (b) $25 \mathrm{mM} \mathrm{Fe}$, (c) $50 \mathrm{mM} \mathrm{Fe}$, (d) $100 \mathrm{mM}$ $\mathrm{Fe}$, and (e) $150 \mathrm{mM}$ Fe. Scale bar $=1 \mu \mathrm{m}$; magnification $40,000 \times$.

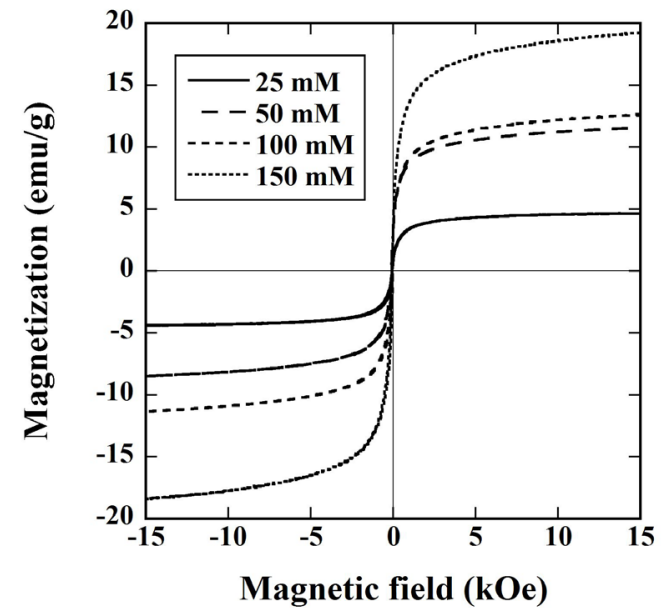

Figure 4. Magnetization of the MNFs with various concentrations of MNPs (25, 50,100 , and $150 \mathrm{mM} \mathrm{Fe}$ ), measured using a vibrating sample magnetometer (VSM). 

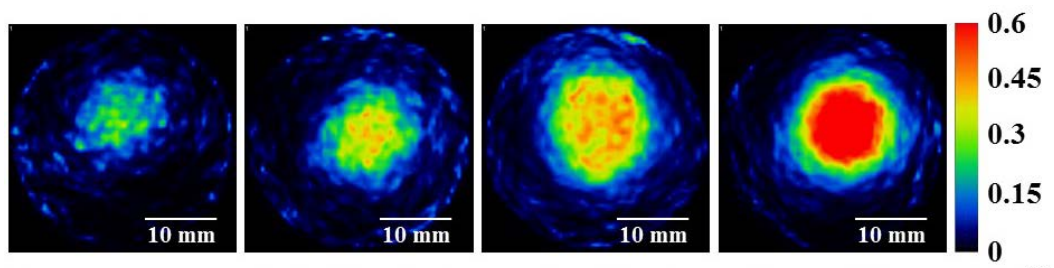

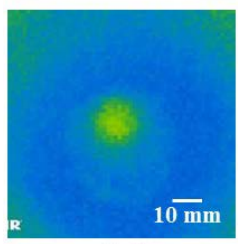

(a)

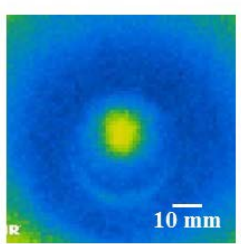

(b)

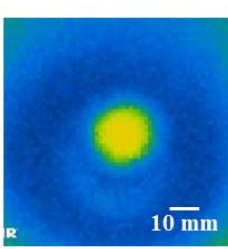

(c)

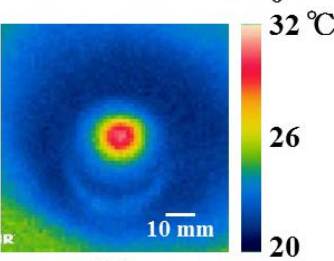

(d)

Figure 5. MPI images of the MNFs (10 $\mathrm{mm}$ in diameter and $2 \mathrm{~mm}$ in thickness) with various concentrations of MNPs (upper row) and the thermal images obtained by an infrared thermometer $10 \mathrm{~min}$ after the start of magnetic hyperthermia (lower row). (a) $25 \mathrm{mM} \mathrm{Fe}$, (b) $50 \mathrm{mM} \mathrm{Fe}$, (c) $100 \mathrm{mM} \mathrm{Fe}$, and (d) $150 \mathrm{mM}$ Fe. Scale bar $=10 \mathrm{~mm}$.
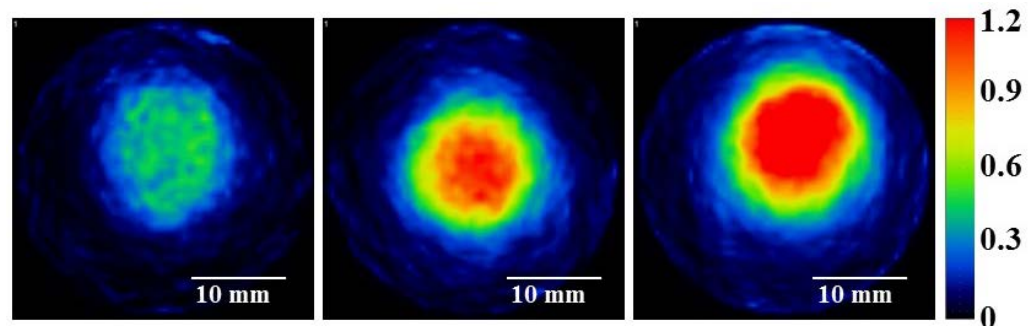

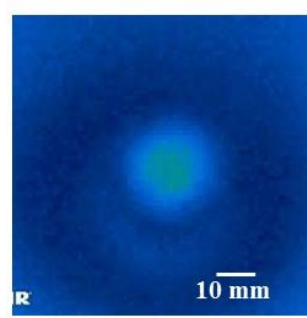

(a)

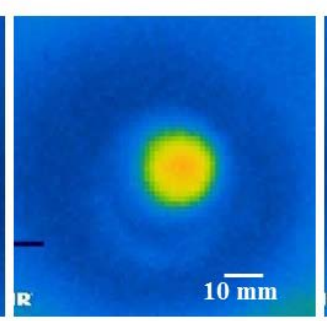

(b)

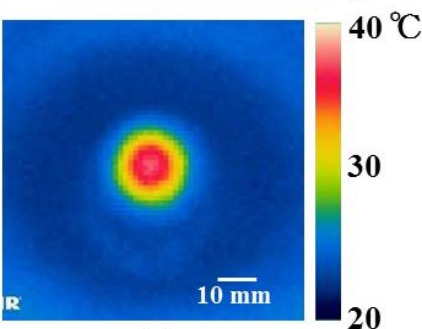

(c)

Figure 6. MPI images of the MNFs (10 $\mathrm{mm}$ in diameter and $2 \mathrm{~mm}$ in thickness) with various numbers of MNF sheets (upper row) and the thermal images obtained by an infrared thermometer $10 \mathrm{~min}$ after the start of magnetic hyperthermia (lower row). (a) one sheet, (b) two sheets, and (c) three sheets. In these cases, the concentration of MNPs was $100 \mathrm{mM}$ Fe. Scale bar $=10 \mathrm{~mm}$.

the start of magnetic hyperthermia also increased with increasing concentration of MNPs (lower row in Figure 5) and increasing number of MNF sheets (lower row in Figure 6).

Figure 7 shows the time courses of the temperature rise in MNFs, which were measured by an infrared thermometer. Figure 7(a) shows the case when the concentration of MNPs was varied as $25 \mathrm{mM}$ (closed circles), 50 $\mathrm{mM}$ (open circles), $100 \mathrm{mM}$ (closed squares), and $150 \mathrm{mM}$ (open squares), whereas Figure 7(b) shows the case when the number of MNF sheets was varied as one (closed circles), two (open circles), and three (closed squares). $(\Delta T / \Delta t)_{0}$ was calculated from Equation (2) by fitting these time courses to Equation (1) (solid lines).

Figures 8(a)-(b) show the relationships between the concentration of MNPs and the average MPI value and between the number of MNF sheets and the average MPI value, respectively. Figures 9(a)-(b) show the relationships between the concentration of MNPs and $(\Delta T / \Delta t)_{0}$ and between the number of MNF sheets and $(\Delta T / \Delta t)_{0}$, respectively. As shown in Figure 8, the average MPI value had excellent correlations with the con- 


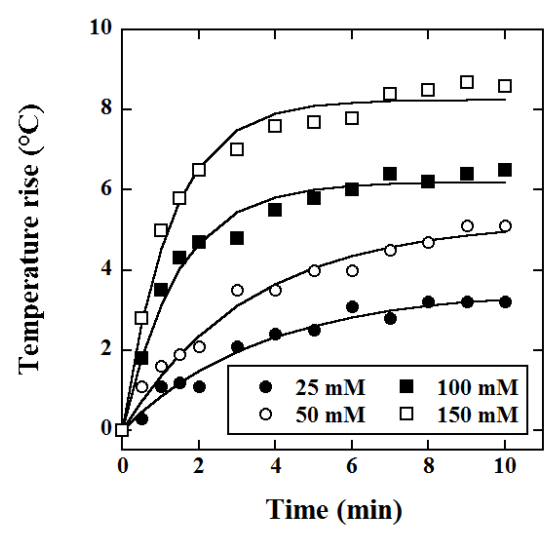

(a)

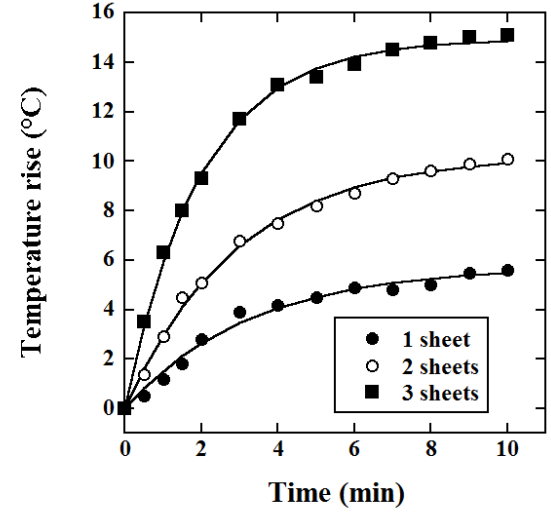

(b)

Figure 7. Time courses of the temperature rise in MNFs, measured by an infrared thermometer. (a) Concentration of MNPs varied as $25 \mathrm{mM}$ Fe (closed circles), $50 \mathrm{mM} \mathrm{Fe}$ (open circles), $100 \mathrm{mM}$ Fe (closed squares), and $150 \mathrm{mM}$ Fe (open squares). (b) Number of MNF sheets varied as one (closed circles), two (open circles), and three (closed squares).

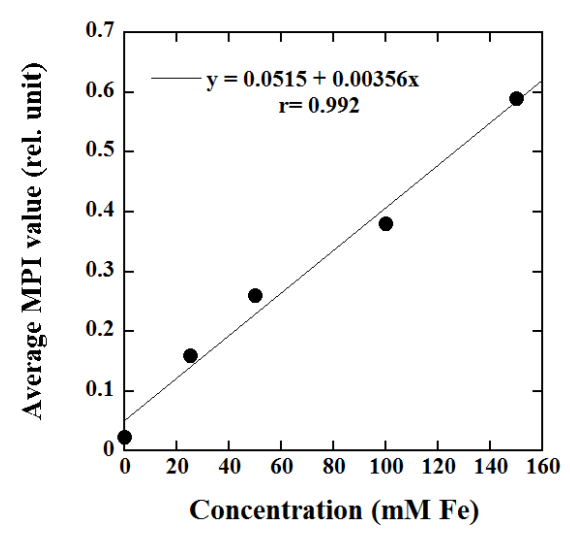

(a)

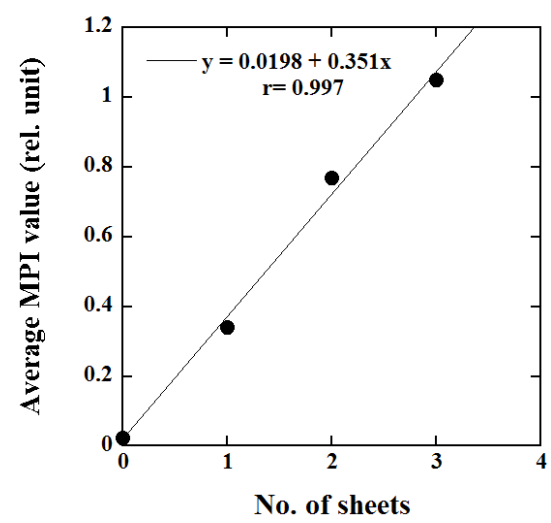

(b)

Figure 8. (a) Relationship between the concentration of MNPs and the average MPI value. (b) Relationship between the number of MNF sheets and the average MPI value.

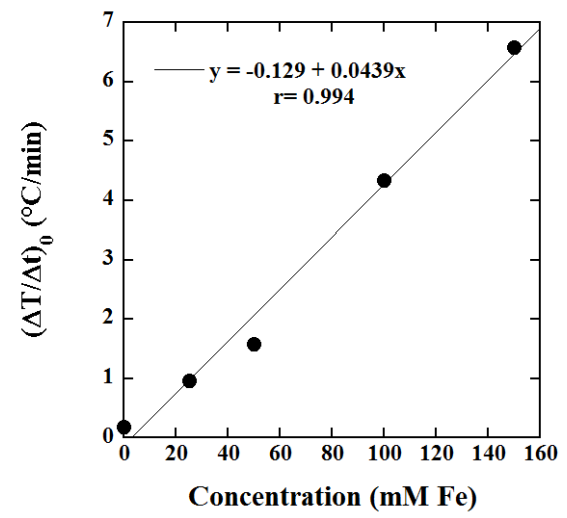

(a)

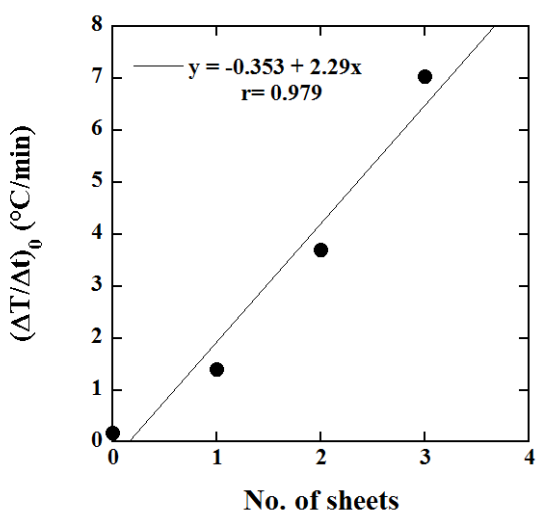

(b)

Figure 9. (a) Relationship between the concentration of MNPs and the initial slope of the time-dependent temperature rise $\left((\Delta T / \Delta t)_{0}\right)$ calculated from Equation (2). (b) Relationship between the number of MNF sheets and $(\Delta T / \Delta t)_{0}$ calculated from Equation (2). 
centration of MNPs $(\mathrm{r}=0.992, P<0.05)$ and the number of MNF sheets $(\mathrm{r}=0.997, P<0.05)$. As shown in Figure 9, $(\Delta T / \Delta t)_{0}$ had also excellent correlations with the concentration of MNPs $(\mathrm{r}=0.994, P<0.05)$ and the number of MNF sheets $(\mathrm{r}=0.979, P<0.05)$.

Figures 10(a)-(c) show the MPI image of the MNFs implanted in the bone of a chicken, the X-ray CT image of the bone in which the MNFs were implanted, and the fusion image between the MPI and X-ray CT images, respectively. As shown in Figure 10(a), MPI could clearly visualize the MNFs implanted in the bone, and the position of the MNFs in the MPI image coincided with that in the X-ray CT image (Figure 10(b)), as confirmed by the fusion image between them (Figure 10(c)).

\section{Discussion}

Chitosan has a cationic linear polysaccharide structure and has great potential for many uses including food, cosmetic, biomedical, and pharmaceutical applications, because it is biocompatible and biodegradable and has cellular-binding, wound-healing, anti-bacterial, and anti-fungal properties [11]. In this study, we developed MNFs by mixing cross-linked chitosan nanofibers with MNPs. This procedure is very simple and easy to implement.

As shown in Figure 3, the SEM images clearly indicated that MNPs aggregated like cocoons on chitosan nanofibers especially when the concentration of MNPs was high. The magnetization measured by VSM (Figure 4) demonstrated that the MNFs developed in this study have a nonlinear response to a magnetic field and the saturation magnetization increases with increasing concentration of MNPs. These magnetic properties appear to be appropriate for MPI to image these MNFs, and the MNFs could be successfully visualized using our MPI scanner (Figure 5, Figure 6, and Figure 10). Furthermore, the average MPI value showed excellent correlation with the concentration of MNPs $(\mathrm{r}=0.992, P<0.05)$ (Figure 8(a)) and the number of MNF sheets $(\mathrm{r}=0.997, P<$ 0.05) (Figure 8(b)), indicating that MPI can quantify the quantity of MNPs in the MNFs.

As shown in Figure 7, a temperature rise was obtained from the MNFs under an alternating magnetic field, suggesting that the MNFs can be used as a source of magnetic hyperthermia. Furthermore, $(\Delta T / \Delta t)_{0}$ showed excellent correlation with the concentration of MNPs $(\mathrm{r}=0.994, P<0.05)$ (Figure 9(a)) and the number of MNF sheets $(\mathrm{r}=0.979, P<0.05)$ (Figure 9(b)). These results suggest that it is possible to perform local temperature control in magnetic hyperthermia by changing the concentration of MNPs in the MNFs and the number or thickness of MNF sheets. Such local temperature control will be important for reducing the side effects and risks of heating surrounding healthy tissues in magnetic hyperthermia.

In addition to the fact that MNFs can be visualized using MPI and can be heated using an alternating magnetic field (Figures 5-10), and that the concentration of MNPs and the number or thickness of MNF sheets can be easily changed, the advantage of the MNFs is that the MNFs can be freely transformed into a desired shape according to the purpose or the shape and/or size of the targeted region, as shown in Figure 10. This aspect also appears to be useful for realizing reliable and safe cancer therapy or tissue regeneration using MNFs.

As previously described, MPI could successfully visualize the MNFs as a positive contrast (Figure 5, Figure 6, and Figure 10). This excellent visibility of MPI appears to be useful for in vivo monitoring of the effective-

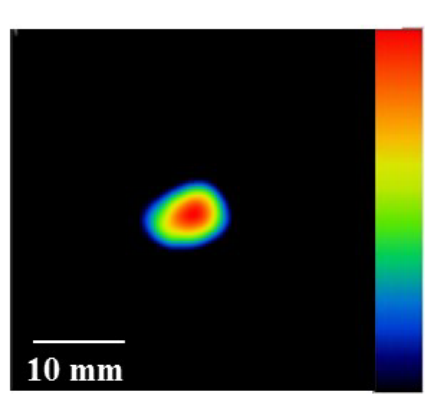

(a)

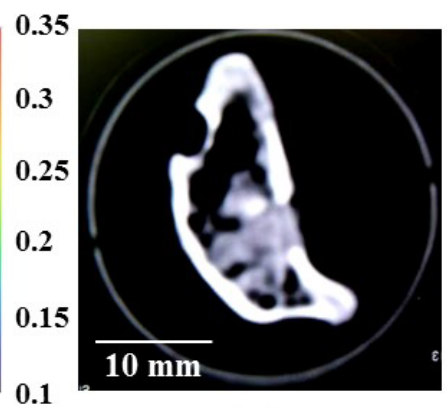

(b)

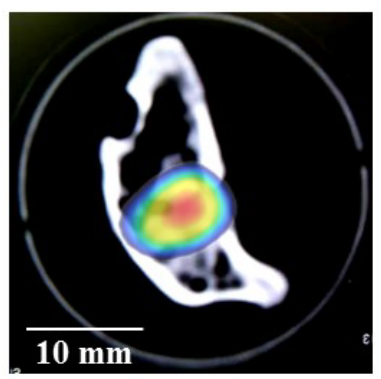

(c)

Figure 10. (a) MPI image of the MNFs (150 mM Fe) implanted in the bone of a chicken, (b) X-ray CT image of the bone implanted with MNFs, and (c) the fusion image between the MPI and X-ray CT images. Scale bar $=10 \mathrm{~mm}$. 
ness of cancer therapy or tissue regeneration using MNFs. Another imaging method to detect MNPs is magnetic resonance imaging (MRI). When we attempted to image MNPs using MRI with a conventional transverse relaxation time $\left(T_{2}^{*}\right)$-weighted imaging sequence, it was almost impossible due to large susceptibility-induced MR signal loss and image distortions in the regions near the MNPs. Recently, however, MRI pulse sequences capable of preserving the signal from spins with ultrashort $T_{2}^{*}$, such as ultrashort echo time (UTE) [17] and sweep imaging with Fourier transformation (SWIFT) sequences [18], have been developed. With these pulse sequences, MNPs can be detected and quantified based on the shortening of the longitudinal relaxation time of water $\left(T_{1}\right)$ [18]. Zhang et al. [19] reported that the reciprocal of $T_{1}$ measured using the SWIFT sequence combined with the Look-Locker method has a linear relationship with MNPs concentration up to $53.6 \mathrm{mM} \mathrm{Fe}$. Although their method also appears to be promising and useful for detecting MNPs, significant research and technology development will be necessary before establishing the effectiveness of their method when translated to animal and/or human studies [20].

Recently, multifunctional nanofibers have been developed by electrospinning polymers mixed with an additional compound such as biomolecules or by surface modification with peptides or proteins [21]. These functional nanofibers are promising for more diverse applications such as drug delivery, tissue engineering, and wound healing [21]. Functionalizing MNFs by mixing with an additional compound or by surface modification would yield more diverse applications of MNFs and MPI might provide a new imaging strategy for monitoring the effectiveness of the functionalized MNFs in these applications.

Although the present study is limited to phantom and ex vivo studies, we will perform in vivo studies using small animals in the near future in order to investigate the feasibility of practical application of the MNFs developed in this study.

\section{Conclusion}

In this study, we developed MNFs by mixing cross-linked chitosan nanofibers with MNPs, and investigated the feasibility of visualizing them using MPI and of heating them using an alternating magnetic field. Our results demonstrate that the MNFs developed in this study can be visualized using MPI and can be used as a source of magnetic hyperthermia, and suggest that MNFs will be useful in biomedicine including cancer therapy and tissue regeneration.

\section{Acknowledgements}

This work was supported by a Grant-in-Aid for Scientific Research (B) (Grant Number: 25282131) from the Japan Society for the Promotion of Science (JSPS).

\section{Declaration of Interest}

The authors report no conflicts of interest. The authors alone are responsible for the content and writing of the paper.

\section{References}

[1] Bock, N., Riminucci, A., Dionigi, C., Russo, A., Tampieri, A., Landi, E., Goranov, A.A., Marcacci, M. and Dediu, V. (2010) A Novel Route in Bone Tissue Engineering: Magnetic Biomimetic Scaffolds. Acta Biomaterialia, 6, 786-796. http://dx.doi.org/10.1016/j.actbio.2009.09.017

[2] Giannoudis, P.V., Einhorn, T.A. and Marsh, D. (2007) Fracture Healing: The Diamond Concept. Injury, 38, S3-S6. http://dx.doi.org/10.1016/S0020-1383(08)70003-2

[3] Meng, J., Xiao, B., Zhang, Y., Liu, J., Xue, H., Lei, J., Kong, H., Huang, Y., Jin, Z., Gu, N. and Xu, H. (2013) Super-Paramagnetic Responsive Nanofibrous Scaffolds under Static Magnetic Field Enhance Osteogenesis for Bone Repair in Vivo. Scientific Reports, 3, Article No. 2655. http://dx.doi.org/10.1038/srep02655

[4] Li, G., Feng, S. and Zhou, D. (2011) Magnetic Bioactive Glass Ceramic in the System $\mathrm{CaO}-\mathrm{P}_{2} \mathrm{O}_{5}-\mathrm{SiO}_{2}-\mathrm{MaO}_{-}-\mathrm{CaF}_{2}-$ $\mathrm{MnO}_{2}-\mathrm{Fe}_{2} \mathrm{O}_{3}$ for Hyperthermia Treatment of Bone Tumor. Journal of Materials Science: Materials in Medicine, 22 , 2197-2206. http://dx.doi.org/10.1007/s10856-011-4417-1

[5] Matsumine, A., Kusuzaki, K., Matsubara, T., Shintani, K., Satonaka, H., Wakabayashi, T., Miyazaki, S., Morita, K., Takegami, K. and Uchida, A. (2007) Novel Hyperthermia for Metastatic Bone Tumors with Magnetic Materials by 
Generating an Alternating Electromagnetic Field. Clinical and Experimental Metastasis, 24, 191-200. http://dx.doi.org/10.1016/j.actbio.2012.03.045

[6] Lin, T.C., Lin, F.H. and Lin, J.C. (2012) In Vitro Feasibility Study of the Use of a Magnetic Electrospun Chitosan Nanofiber Composite for Hyperthermia Treatment of Tumor Cells. Acta Biomaterialia, 8, 2704-2711. http://dx.doi.org/10.1016/j.actbio.2012.03.045

[7] Gleich, B. and Weizenecker, J. (2005) Tomographic Imaging Using the Nonlinear Response of Magnetic Particles. Nature, 435, 1214-1217. http://dx.doi.org/10.1038/nature03808

[8] Goodwill, P.W., Konkle, J.J., Zheng, B., Saritas, E.U. and Conolly, S.M. (2012) Projection X-Space Magnetic Particle Imaging. IEEE Transactions on Medical Imaging, 31, 1076-1085. http://dx.doi.org/10.1109/TMI.2012.2185247

[9] Murase, K., Hiratsuka, S., Song, R. and Takeuchi, Y. (2014) Development of a System for Magnetic Particle Imaging Using Neodymium Magnets and Gradiometer. Japanese Journal of Applied Physics, 53, Article ID: 067001. http://dx.doi.org/10.7567/JJAP.53.067001

[10] Murase, K., Song, R. and Hiratsuka, S. (2014) Magnetic Particle Imaging of Blood Coagulation. Applied Physics Letters, 104, Article ID: 252409. http://dx.doi.org/10.1063/1.4885146

[11] Dutta, A.K., Kawamoto, N., Sugino, G., Izawa, H., Morimoto, M., Saimoto, H. and Ifuku, S. (2013) Simple Preparation of Chitosan Nanofibers from Dry Chitosan Powder by the Start Burst System. Carbohydrate Polymers, 97, 363367. http://dx.doi.org/10.1016/j.carbpol.2013.05.010

[12] Murase, K., Konishi, T., Takeuchi, Y., Takata, H. and Saito, S. (2013) Experimental and Simulation Studies on the Behavior of Signal Harmonics in Magnetic Particle Imaging. Radiological Physics and Technology, 6, 399-414. http://dx.doi.org/10.1007/s12194-013-0213-6

[13] Murase, K., Banura, N., Mimura, A. and Nishimoto, K. (2015) Simple and Practical Method for Correcting the Inhomogeneous Sensitivity of a Receiving Coil in Magnetic Particle Imaging. Japanese Journal of Applied Physics, 54, Article ID: 038001. http://dx.doi.org/10.7567/JJAP.54.038001

[14] Murase, K., Oonoki, J., Takata, H., Song, R., Angraini, A., Ausanai, P. and Matsushita, T. (2011) Simulation and Experimental Studies on Magnetic Hyperthermia with Use of Superparamagnetic Iron Oxide Nanoparticles. Radiological Physics and Technology, 4, 194-202. http://dx.doi.org/10.1007/s12194-011-0123-4

[15] Box, G.E.P. and Lucas, H.L. (1959) Design of Experiments in Nonlinear Situations. Biometrika, 46, 77-90. http://dx.doi.org/10.1093/biomet/46.1-2.77

[16] Murase, K., Takata, H., Takeuchi, Y. and Saito, S. (2013) Control of the Temperature Rise in Magnetic Hyperthermia with Use of a Static Magnetic Field. Physica Medica, 29, 624-630. http://dx.doi.org/10.1016/j.ejmp.2012.08.005

[17] Robson, M.D., Gatehouse, P.D., Bydder, M. and Bydder, G.M. (2003) Magnetic Resonance: An Introduction to Ultrashort TE (UTE) Imaging. Journal of Computer Assisted Tomography, 27, 825-846. http://dx.doi.org/10.1097/00004728-200311000-00001

[18] Idiyatullin, D., Corum, C., Park, J.Y. and Garwood, M. (2006) Fast and Quiet MRI Using a Swept Radiofrequency. Journal of Magnetic Resonance, 181, 342-349. http://dx.doi.org/10.1016/j.jmr.2006.05.014

[19] Zhang, J.J., Chamberlain, R., Etheridge, M., Idiyatullin, D., Corum, C., Bischof, J. and Garwood, M. (2014) Quantifying Iron-Oxide Nanoparticles at High Concentration Based on Longitudinal Relaxation Using a Three-Dimensional SWIFT Look-Locker Sequence. Magnetic Resonance in Medicine, 71, 1982-1988. http://dx.doi.org/10.1002/mrm.25181

[20] Hoopes, P.J., Petryk, A.A., Tate, J.A., Savellano, M.S., Strawbridge, R.R., Giustini, A.J., Stan, R.V., Gimi, B. and Garwood, M. (2013) Imaging and Modification of the Tumor Vascular Barrier for Improvement in Magnetic Nanoparticle Uptake and Hyperthermia Treatment Efficacy. Proceedings SPIE Energy-Based Treatment of Tissue and Assessment VII, 8584, Article ID: 858403. http://dx.doi.org/10.1117/12.2008689

[21] Kim, Y.J., Ebara, M. and Aoyagi, T. (2013) A Smart Hyperthermia Nanofiber with Switchable Drug Release for Inducing Cancer Apoptosis. Advanced Functional Materials, 23, 5753-5761. http://dx.doi.org/10.1002/adfm.201300746 